\title{
Actualización en el diagnóstico y tratamiento de la acidemia isovalérica.
}

Revisión

Ivonne Martín-Hernández.

Laboratorio de Errores Innatos del Metabolismo. Centro Nacional de Genética Médica, La Habana, Cuba.

\section{RESUMEN}

Objetivos. Este trabajo ofrece una revisión teórica y actualizada sobre la acidemia isovalérica, dirigida no sólo al personal médico especializado en este tema, sino también a otros especialistas interesados en el estudio de los errores innatos del metabolismo.

Fuentes de extracción. Artículos actualizados y libros de texto relacionados con el tema. Consulta en Internet.

Resultados. La acidemia isovalérica es un error congénito autosómico recesivo del catabolismo de la leucina causado por la deficiencia de la isovaleril coenzima A deshidrogenasa. Esta deficiencia enzimática conlleva a un desorden metabólico severo, manifestado clínicamente como vómitos, deshidratación, acidosis, convulsiones, coma y muerte. Fundamentalmente, el diagnóstico de este desorden consiste en la medición de diferentes metabolitos en sangre y orina, y en el análisis de la actividad enzimática. Con detección temprana el pronóstico y calidad de vida mejora grandemente. El tratamiento consiste en una dieta baja en proteínas, fórmulas especializadas que no contengan leucina, y suplementos de carnitina y/ó glicina.

Conclusiones. Debido a lo raro de este desorden las áreas de salud y los médicos tienen relativamente poca experiencia en el manejo del mismo. Se hace vital la estrecha relación de trabajo entre los clínicos y el laboratorio para el diagnóstico temprano, la pronta transferencia a un centro especializado y una mejor atención a la salud de nuestra población pediátrica.

(Rev Biomed 2006; 17:213-223)

Palabras clave: acidemia isovalérica, errores congénitos del metabolismo, diagnóstico, tratamiento.

\section{SUMMARY.}

Update on the diagnosis and treatment of isovaleric acidemia.

Objectives. This work offers a theoretical and updated review about isovaleric acidemia, directed not only to medical specialists in this topic, but also to other specialists interested in the knowledge of inborn errors of metabolism.

Solicitud de sobretiros: Lic. Ivonne Martín-Hernández. Felipe Poey\# 54 el. Luis Estévez y Lacret. Rpto, Santos Suárez, Municipio 10 de Octubre, Ciudad de la Habana, Cuba. Correo electrónico: ivonne.martin@infomed.sld.cu Recibido el 5/Enero/2006. Aceptado para publicación el 27/Junio/2006. 


\section{Martín-Hernández}

Sources of information. Updated articles and books related to the theme. Internet search.

Results. Isovaleric acidemia is a rare autosomal recessive inborn error of leucine catabolism caused by deficiency of isovaleryl coenzyme A dehydrogenase. This enzymatic deficiency leads to severe metabolic derangement, manifested clinically as vomiting, dehydration, acidosis, seizures, coma, and death. Fundamentally, the diagnosis of this disorder consists of the measurement of various metabolites in blood and urine, and in enzyme activity analysis. With early detection the prognosis and quality of life improves greatly. Treatment consists of a low-protein diet, a specialized formula that contains no leucine, and supplements of carnitine and/or glycine.

Conclusions. Because it is rare, health care personnel and physicians have relatively little experience in dealing with this disorder. A tighter work relationship between clinicians and laboratory staff for an early diagnosis, prompt referral to a specialized center, and a better health service for our pediatric population is vital.

(Rev Biomed 2006; 17:213-223)

Key words: Isovaleric acidemia, inborn errors of metabolism, diagnosis, treatment.

\section{INTRODUCCIÓN.}

Los errores congénitos del metabolismo son enfermedades monogénicas donde la alteración de un gen produce un defecto enzimático que conduce a las alteraciones bioquímicas características de cada enfermedad metabólica y son responsables de los fenotipos desadaptativos propios de cada patología. Específicamente, las deficiencias enzimáticas conocidas en los distintos pasos del catabolismo de los aminoácidos se conocen con el nombre genérico de acidurias orgánicas. Éstas conllevan a la acumulación de determinados metabolitos, generalmente tóxicos, y a la deficiencia de otros, indispensables para el funcionamiento normal del organismo. Cuando se presentan durante el período neonatal, lo hacen como enfermedades devastadoras que ponen en peligro la vida del niño, lo cual hace esencial un diagnóstico adecuado y rápido, y tratamientos específicos y de soporte (1).

En la actualidad se conocen seis desórdenes heredables que involucran la vía catabólica de la leucina, considerados acidurias orgánicas de cadena ramificada. Entre los más estudiados están: acidemia isovalérica, deficiencia de 3-metilcrotonil-CoA carboxilasa, aciduria 3metilglutacónica y deficiencia de 3-hidroxi-3metilglutaril CoA liasa. De ellas, la acidemia isovalérica (AIV) es la más frecuente. Figura 1.

La herencia de la AIV es autosómica recesiva y su incidencia se desconoce debido a la carencia de estudios de tamiz en población general. Esta patología ha sido identificada en varios grupos étnicos y raciales. Su nombre deriva de las elevadas concentraciones de ácido isovalérico encontrado en los pacientes con dicha patología (2).

\section{BIOQUÍMICA Y GENÉTICA MOLECULAR.}

La AIV es causada por el déficit en la actividad enzimática de la apoenzima mitocondrial isovalerilCoA deshidrogenasa. La existencia de esta enzima fue propuesta en el año 1966 por Tanaka y colaboradores. Se conoce que es una flavoproteína que transfiere electrones a la cadena respiratoria por medio de la flavoproteína transportadora de electrones (FTE) y que corresponde a la tercera enzima de la vía de degradación de la leucina. Esta enzima metaboliza el isovaleril-CoA a 3metilcrotonil-CoA y tiene actividad en hígado, leucocitos y fibroblastos humanos. En su defecto se produce un acúmulo intracelular de isovalerilCoA con la aparición de metabolitos característicos en los pacientes afectados (1-4).

La isovaleril-CoA deshidrogenasa es sintetizada en los polisomas citosólicos como una subunidad precursora de $45 \mathrm{kDa}$, que posteriormente es procesada a una subunidad de 43 kDa durante su importación a la mitocondria donde cuatro subunidades idénticas forman una enzima tetramérica de $172 \mathrm{kDa}$ localizada en la matriz o

\section{Revista Biomédica}


Acidemia isovalérica

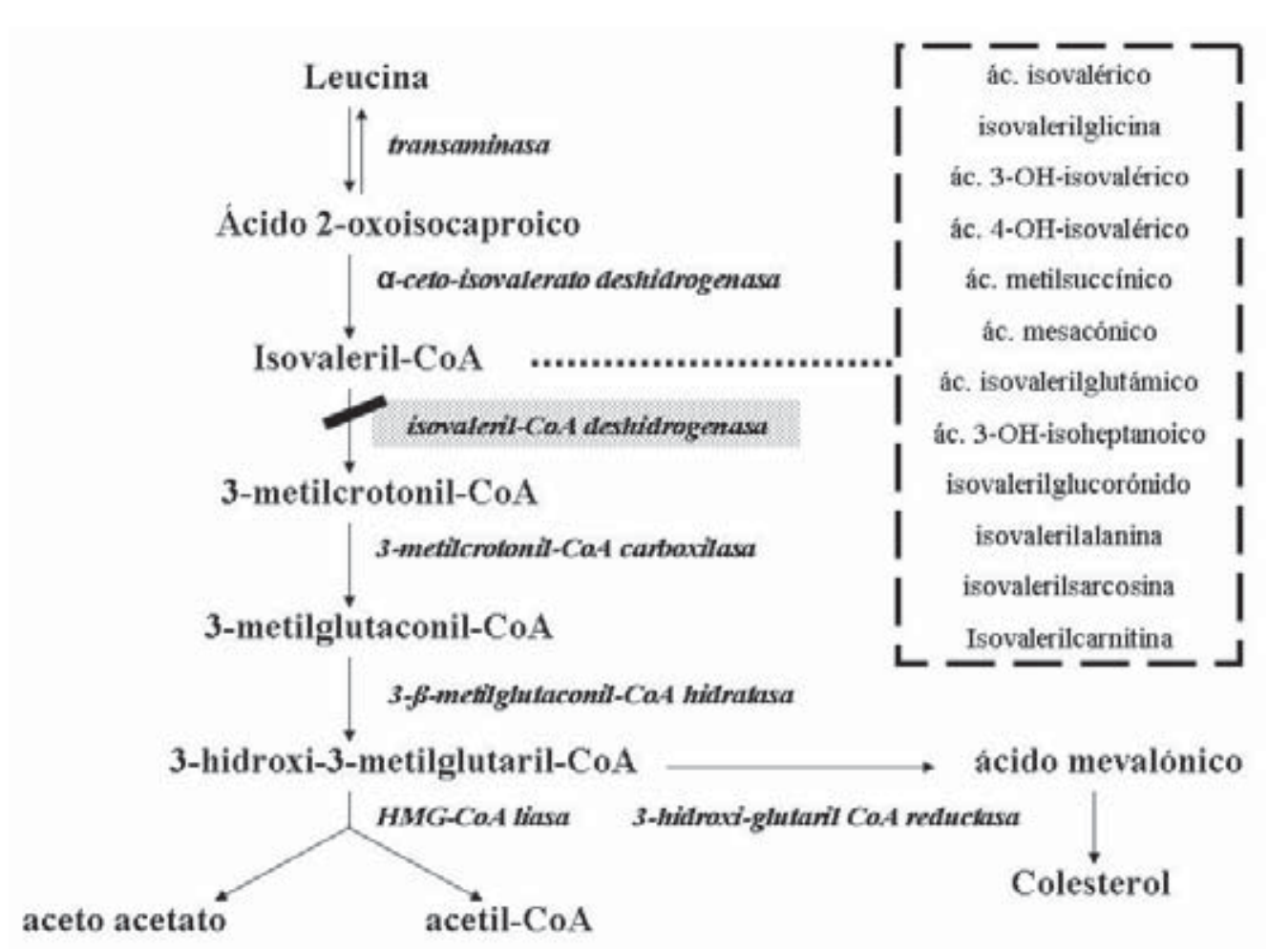

Figura 1.- Vía catabólica del aminoácido esencial leucina.

en la cara interna de la membrana mitocondrial (5, 6).

El gen que codifica para la enzima isovalerilCoA deshidrogenasa se encuentra localizado en el cromosoma 15q14-q15 y contiene 12 exones. Hasta la fecha se conocen ocho mutaciones génicas (2, 7-11).

\section{SINTOMATOLOGÍA BIOQUÍMICA Y} CLÍNICA.

La acumulación de ácidos orgánicos produce una serie de síntomas bioquímicos característicos en los pacientes con AIV como: acidosis $\left(\mathrm{pH}<7.5 ; \mathrm{HCO}_{3}<15\right)$, glucosa normal o elevada, hiperlactatemia $(>3.5 \mathrm{mmol} / \mathrm{L})$, hiperamonemia (hasta $>500 \mu \mathrm{mol} / \mathrm{L}$ ), calcio normal o ligeramente disminuido y cetonemia/cetonuria $(12,13)$.

Han sido reportadas tres formas de presentación clínica de la AIV: una forma aguda neonatal, una forma crónica intermitente y una forma lentamente progresiva (12). Estas diferentes formas pueden ocurrir en una misma familia, por lo que no están relacionadas al genotipo.

En la presentación aguda neonatal los síntomas aparecen en un recién nacido de término sin causa aparente, después de un período sin síntomas que pueden ser de pocas horas o días (de 1 a 14 días, usualmente de 3 a 6 días) junto a una grave cetoacidosis metabólica inexplicada. Se trata de síntomas inespecíficos de intoxicación que no responden a medicación habitual, presentando rechazo de la alimentación, succión débil, vómitos, deshidratación y pérdida de peso. Aparecen síntomas de disfunción del sistema nervioso central (SNC) debido al acúmulo de ácidos orgánicos y amonio, con letargia, hipotonía, temblores y convulsiones. La asociación de vómitos, distensión abdominal y constipación puede sugerir una obstrucción intestinal. El bebé sin causa aparente inicia además dificultad respiratoria, bradicardia, apneas e hipotermia, pudiendo llegar al coma y muerte. Además se hace característico un olor repugnante a "pie sudado" o a "queso". Las alteraciones bioquímicas más frecuentes encontradas son hipo/ hiperglicemia, cetoacidosis con aumento del anión gap, hiperamonemia, hiperglicinemia, aumento

Vol. 17/No. 3/Julio-Septiembre, 2006 


\section{Martín-Hernández}

de ácido láctico, cetonuria, y anemia. En estos pacientes se encuentran deprimidas las funciones de la médula ósea conllevando a neutropenia, leucopenia, trombocitopenia y pancitopenia lo que conduce a infecciones y/o hemorragia cerebral (2, 4, 12-16).

En la forma crónica intermitente los primeros episodios de la enfermedad usualmente ocurren durante el primer año de vida o tardíamente en la infancia. Los episodios intermitentes se deben a menudo a infecciones respiratorias o ingestión de comidas ricas en proteínas, estrés o a motivos desconocidos, y se resuelven con restricción de proteínas e infusión de glucosa. Se suelen presentar con síntomas digestivos como vómitos cíclicos acetonémicos, diarrea, gastroenteritis, retardo pondoestatural, afectación del estado general, aversión a proteínas y complicaciones neurológicas como depresión sensorial con letargia progresiva que puede evolucionar al coma, y sintomatología extrapiramidal. Es común hallar una acidosis metabólica en ocasiones con hiperamonemia, cetonuria, trombocitopenia, neutropenia y pancitopenia. En algunos casos se presenta alopecia e hiperglicemia. La mayoría de estos pacientes tienen desarrollo psicomotor normal, otros en cambio presentan hipotonía y retardo del crecimiento y desarrollo (2, 4, 12-14, 17-19).

La forma lentamente progresiva se caracteriza por anorexia persistente, síntomas digestivos, retardo en el desarrollo pondoestatural y psicomotor, osteoporosis, sintomatología extrapiramidal/piramidal e incluso deterioro mental progresivo. Algunas de estas formas son oligosintomáticas (vértigo intermitente, ataxia truncal o trastornos de visión); excepcionalmente pueden permanecer totalmente asintomáticos. Se han descrito en pacientes complicaciones clínicas como pancreatitis $(12,20)$.

\section{METABOLITOS ANORMALES.}

El ácido isovalérico es el compuesto que les confiere el olor a "pie sudado" a los pacientes con AIV. Durante la remisión de los pacientes la concentración del ácido isovalérico en plasma puede tener valor normal (menor que $10 \mu \mathrm{M}$ ) o hasta diez veces su valor, pero durante los episodios severos los niveles alcanzan de cien a quinientas veces las concentraciones normales ( 600 a 5000 $\mu \mathrm{M})$. Las cantidades de ácido isovalérico en orina de pacientes es mucho menor que en plasma por ser muy volátil y los rangos de excreción son de 8 a $300 \mu \mathrm{mol} / \mathrm{día}$ (normales: menos de $2 \mu \mathrm{mol} / \mathrm{día}$ ) $(2,3)$.

El mayor metabolito producto del isovalerilCoA debido a la deficiencia de la isovaleril-CoA deshidrogenasa es la isovalerilglicina. Esta reacción la cataliza la enzima mitocondrial glicina N-acilasa. Los niveles de excreción de isovalerilglicina en pacientes con AIV alcanzan 2000 a $15000 \mu \mathrm{mol} / \mathrm{día}$ (valores normales $<15$ $\mu \mathrm{mol} /$ día). Esta excreción es mayor durante los episodios agudos, pero permanecen muy altas durante la remisión (1 000 a $3000 \mathrm{mmol} / \mathrm{mol}$ de creatinina), aunque parece no ser tóxica y constituye el único metabolito diagnóstico en este momento $(2,12,21)$.

Otro compuesto excretado en cantidades anormales durante los episodios agudos es el ácido 3-hidroxi-isovalérico. Este ácido alcanza cifras de hasta $3000 \mu \mathrm{mol} / \mathrm{día}$ y se piensa provenga de la $\omega$-oxidación del elevado ácido isovalérico libre $(2,22,23)$.

Un gran número de metabolitos adicionales del isovaleril-CoA han sido identificados en la orina de pacientes con AIV como: isovalerilcarnitina, isovalerilglucorónido y ácido 4-hidroxi-isovalérico (2, 24-27). El ácido 4-hidroxi-isovalérico puede ser posteriormente oxidado a ácido 2 metil-succínico y entonces deshidrogenado a ácido 2-metilfumárico (ácido mesacónico). El isovaleril-CoA puede ser también condensado con acetil-CoA por la enzima 3-oxotiolasa para formar ácido 3-hidroxi-isoheptanoico. También son formados como productos de la conjugación del isovalerilCoA los compuestos orgánicos siguientes: isovalerilalanina, isovalerilsarcosina y ácido isovalerilglutámico (2, 26-28). Estos metabolitos

\section{Revista Biomédica}


pueden estar elevados durante episodios agudos en los pacientes con AIV ( 20 a $300 \mathrm{mmol} / \mathrm{mol} \mathrm{de}$ creatinina), pero de todos ellos solamente el ácido isovalerilglucorónido y la isovalerilcarnitina son de importancia y sus excreciones son usualmente una pequeña fracción en comparación con la isovalerilglicina. Es de importancia diagnóstica la elevación de la isovalerilcarnitina en plasma.y sangre seca sobre papel de filtro (2).

En muy pocos pacientes se ha encontrado ligera elevación de los niveles en plasma del aminoácido aloisoleucina (2 a $10 \mu \mathrm{M})$ (29).

Los investigadores de este campo se han dado a la tarea de seguir la búsqueda de metabolitos que los ayuden en el entendimiento y tratamiento de este desorden. Recientemente se han hallado en el perfil urinario de estos pacientes 19 metabolitos conjugados isovaleril-aminoácidos y acetilaminoácidos. Estos son:

Isovaleril-conjugados de los aminoácidos: ácido $\alpha$-aminobutírico, ácido aspártico, serina, fenilalanina, treonina, valina, leucina, tirosina, asparagina, histidina, lisina y triptófano.

Conjugados acetilados de los aminoácidos: treonina, valina, glicina, $\beta$-alanina, $\alpha$-alanina, tirosina y triptófano (30).

\section{DIAGNÓSTICO Y PRONÓSTICO.}

La sintomatología clínica como el olor a "pie sudado" durante los episodios agudos permite a los médicos una orientación diagnóstica en este tipo de patología, pero generalmente este olor no se presenta durante remisión y no siempre es notable durante los episodios. Otras patologías como la acidemia glutárica tipo II presenta olores similares debido a la acumulación de ácido butírico, isobutírico, 2-metilbutírico e isovalérico (2).

Debido a que las acidurias orgánicas comparten rasgos clínicos comunes, para un diagnóstico discriminatorio se requiere del análisis de los ácidos orgánicos. La búsqueda de los metabolitos anormales en los fluidos biológicos y la cuantificación de los constituyentes normales (pero modestamente elevados), permiten sugerir un diagnóstico de AIV y se realizan fundamentalmente empleando la técnica de cromatografía de gases acoplada a espectrometría de masas (31).

La cuantificación de carnitina libre y esterificada complementa al análisis de ácidos orgánicos para el diagnóstico de AIV, encontrándose disminución de carnitina libre en suero con aumento de isovalerilcarnitina en suero y orina. Durante la remisión los niveles de isovalerilcarnitina disminuyen hasta 10$20 \mathrm{mmol} / \mathrm{mol}$ de creatinina. Para confirmar el diagnóstico de AIV mediante la cuantificación de isovalerilcarnitina en orina, usualmente se administra oralmente $100 \mathrm{mg} / \mathrm{kg}$ de carnitina haciendo que se alcancen valores de hasta 3200 $\mathrm{mmol} / \mathrm{mol}$ de creatinina $(32,33)$.

La espectrometría de masas tándem ha sido útil para la detección de carnitina e isovalerilcarnitina (aunque esta técnica no distingue entre los diferentes isómeros: 2-metilbutirilcarnitina, isobutirilcarnitina y butirilcarnitina). Esta técnica tiene la ventaja de permitir un diagnóstico temprano en recién nacidos de esta aciduria orgánica, al poder ser utilizadas las muestras de sangre seca sobre papel de filtro recogidas para el cribado neonatal rutinario (2, 31, 34-37).

La resonancia magnética nuclear protónica de alto campo es una técnica que se emplea para un rápido diagnóstico de AIV, mediante la cual se detecta isovalerilglicina en una pequeña alícuota de orina (38, 39).

Entre otros exámenes bioquímicos se recomienda la cuantificación de aminoácidos en plasma y orina, siempre teniendo presente que un perfil normal no excluye el diagnóstico. En los pacientes con AIV se encuentra una ligera elevación de glicina, y cistinuria-lisinuria (12).

La mayoría de los conjugados isovaleril- y acetil-aminoácidos son detectados y cuantificados mediante la técnica cromatografía de gases acoplada a espectrometría de masas, salvo los conjugados de los aminoácidos triptófano, lisina, histidina y asparagina (debido a sus características polares), que se identifican y cuantifican por

Vol. 17/No. 3/Julio-Septiembre, 2006 


\section{Martín-Hernández}

espectrometría de masas tándem (30).

La deficiencia de la isovaleril-CoA deshidrogenasa en fibroblastos puede ser confirmada por ensayos fluorimétricos y liberación de titrio del $\left[2,3-{ }^{3} \mathrm{H}\right]$-isovaleril-CoA, aunque desafortunadamente estos aún no están disponibles (2, 40, 41). Otros métodos utilizados son: determinación de la eficiencia del catabolismo de la leucina por oxidación de $\left[2-{ }^{14} \mathrm{C}\right]$ de la leucina a ${ }^{14} \mathrm{CO}_{2}$, la incorporación de $\left[1-{ }^{14} \mathrm{C}\right]$ del ácido isovalérico a proteínas ambos en cultivos de fibroblastos y la oxidación de $\left[1-{ }^{14} \mathrm{C}\right]$ del ácido isovalérico a ${ }^{14} \mathrm{CO}_{2}$ en leucocitos $(3,12,42)$. Aunque el diagnóstico de portadores es posible mediante los estudios enzimáticos, no se han identificado portadores (2).

Ha sido reportada la determinación de la actividad enzimática en pacientes con AIV mediante la determinación del 3-metilcrotonilCoA a través de la técnica de cromatografía líquida de alta resolución (HPLC). Este método promete ser útil como prueba confirmatoria para los casos de $A I V$ detectados a través del tamizaje por tándem en recién nacidos (43).

Más de la mitad de los pacientes reportados con la forma neonatal aguda han muerto. La mayoría de los pacientes con la forma crónica intermitente tienen un desarrollo psicomotor normal, aunque algunos tienen retraso del desarrollo y retraso mental leve, o incluso severo.

La posibilidad de un pronóstico favorable con un desarrollo normal en los pacientes con AIV, ha mejorado considerablemente cuando se combinan el diagnóstico temprano con un tratamiento adecuado $(2,44)$.

\section{DIAGNÓSTICO PRENATAL.}

Para este tipo de patología es posible el diagnóstico prenatal en el primer y segundo trimestre, el cual puede llevarse a cabo mediante diferentes técnicas como: ensayos fluorimétricos para la determinación de la actividad enzimática en las vellosidades coriónicas, incorporación de [1${ }^{14} \mathrm{C}$ ] del ácido isovalérico en cultivo de amniocitos y cuantificación de isovalerilglicina en líquido amniótico mediante el análisis de dilución de isótopos estables. El análisis de isovalerilglicina en la orina materna no es un método de análisis conveniente. Un nuevo método utilizado para diagnóstico prenatal de AIV es el análisis de isovalerilcarnitina en líquido amniótico mediante espectrometría de masas tándem (2, 12, 45-47).

\section{TRATAMIENTO.}

A los pacientes con AIV durante los episodios agudos y descompensaciones agudas se les indica un tratamiento que consiste en:

a. Suprimir el aporte proteico durante 24 a 48 horas hasta que el amonio sea inferior a $80 \mu \mathrm{mol} /$ L, a partir de entonces se reintroducen proteínas sin leucina (hasta 2 g/kg/día) y se comprueba tolerancia.

b. Simultáneamente, mantener el aporte calórico, mantener hidratación y forzar diuresis con soluciones glucosadas al $10 \%$ o $15 \%$ con electrolitos (100-150 ml/kg/día), vía intravenosa.

c. Administración de lípidos (0,5-4 g/kg/día), L-glicina (150 a 600 mg/kg/día) vía enteral y Lcarnitina (100 a $400 \mathrm{mg} / \mathrm{kg} /$ día), endovenosa si es preciso.

d. Aportar bicarbonato para corregir acidosis si pH sanguíneo es menor de 7.20.

e. Tratamiento de las infecciones intercurrentes.

f. Si es necesario, soporte circulatorio, intubación y ventilación asistida.

g. Para evitar catabolismo proteico puede ser de ayuda la insulina y/o la alanina.

$h$. En pacientes graves puede ser necesario el uso de procedimientos rápidos de eliminación de toxinas (exanguinotransfusión, diálisis o hemofiltración) y/o alimentación parenteral. (2, 4, 12, 48-52).

Es importante tener en cuenta que son causas de descompensaciones agudas las infecciones, inmunizaciones, fiebre de cualquier origen, cambios de dieta, anorexia, vómitos, diarrea y cirugía. Los síntomas de descompensación más comunes son: anorexia, disminución de la actividad con tendencia al sueño, hipotonía,

\section{Revista Biomédica}


equilibrio inestable y polipnea (12).

El tratamiento nutricional crónico se comienza una vez establecido el diagnóstico definitivo. Este deberá ser individualizado en función de cada paciente, la tolerancia, respuesta a los cofactores, estado nutricional y las descompensaciones. En él se indica la restricción de proteínas de la dieta natural a 1.2 a $1.5 \mathrm{~g} / \mathrm{kg} /$ día en lactantes y en niños mayores a $1 \mathrm{~g} / \mathrm{kg} / \mathrm{día}$, lo que implica aportar sólo el $60 \%$ de las recomendaciones entregadas por organismos internacionales, requiriéndose el uso de fórmulas especiales sin leucina, que aportan proteínas de buena calidad y promueven el anabolismo como la línea Leu (Milupa) y la línea Xleu (SHS). La introducción de leucina se inicia cuando su nivel en sangre está entre 50 y $100 \mu \mathrm{M} / \mathrm{L}$, con leche humana o maternizada. Posteriormente las cantidades se van ajustando según nivel de leucina y crecimiento. Un nivel de leucina por debajo de $50 \mu \mathrm{M} / \mathrm{L}$ provoca desbalance de aminoácidos neutros, producido por una restricción mayor de proteínas naturales y una excesiva cantidad de fórmula sin leucina. Este desbalance provoca riesgo de descompensación metabólica, pérdida de cabello y peso, rash en piel, inapetencia y apatía. Se debe mantener un aporte suficiente de aminoácidos esenciales y no esenciales. Se debe aumentar el aporte de proteínas naturales a $1.5 \mathrm{~g} / \mathrm{kg} /$ día y mantener suplementación de L-glicina y L-carnitina (2, 4, 12, 48, 49, 52, 53). Cuadro1.

El ácido isovalérico se conjuga tanto con glicina como con carnitina para ser excretado por la orina. Esto tiene interés terapéutico, puesto que tanto la prescripción de glicina como de carnitina transforman el ácido isovalérico que es neurotóxico en compuestos que no lo son (isovalerilglicina e isovalerilcarnitina).

Las dosis indicadas de glicina son 150-300 $\mathrm{mg} / \mathrm{kg} / \mathrm{d}$ á, aportándose según la edad, estado clínico, porcentaje de actividad enzimática residual e ingesta del aminoácido leucina. El nivel plasmático de glicina se debe mantener entre los 200 y $500 \mu \mathrm{mol} / \mathrm{L}$. Los compuestos como el ácido benzoico y el ácido salicílico compiten con el isovaleril-CoA por la enzima glicina $\mathrm{N}$-acilasa, interfiriendo con el efecto beneficioso de la glicina por lo que son contraindicados en pacientes con $\operatorname{AIV}(2,4,12,48,52-55)$.

Muchos pacientes con AIV hacen deficiencia secundaria de carnitina porque el ácido isovalérico se une a esta amina formando la isovalerilcarnitina, la cual aparece elevada en plasma y orina. La suplementación con 100 a 300 mg/kg/día, dosificada 3 veces al día, previene la deficiencia y ayuda a la exfoliación de este ácido orgánico. El

\section{Cuadro 1}

Recomendación de nutrientes en acidemia isovalérica

\begin{tabular}{cccc}
\hline $\begin{array}{c}\text { Edad } \\
\text { (meses) }\end{array}$ & $\begin{array}{c}\text { Leucina } \\
\mathbf{( m g / k g )}\end{array}$ & $\begin{array}{c}\text { Proteína } \\
\mathbf{( g / k g )}\end{array}$ & $\begin{array}{c}\text { Calorías } \\
\mathbf{( k c a l / k g )}\end{array}$ \\
\hline \hline $0-3$ & $80-150$ & $3-3,5$ & 120 \\
$3-6$ & $70-140$ & $3-3,5$ & 115 \\
$6-9$ & $60-130$ & $2,5-3$ & 110 \\
$9-12$ & $50-120$ & $2,5-3$ & 105 \\
\hline \hline años & $\mathbf{m g} /$ día & g/día & kcal/día \\
$1-4$ & $500-600$ & $>30$ & 1300 \\
$4-7$ & $600-900$ & $>35$ & 1700 \\
$7-11$ & $700-900$ & $>45$ & 2400 \\
\hline \hline
\end{tabular}




\section{Martín-Hernández}

nivel de carnitina libre sobre $35 \mu \mathrm{M} / \mathrm{L}$ indica un buen control; un nivel inferior provoca aumento del ácido 3-hidroxi-isovalerato y se debe aumentar su aporte diario y verificar la causa. Estos pacientes hacen anorexia debido al aumento de síntesis de serotonina por incremento del paso del triptófano a través de la barrera hematoencefálica $(2,4,12$, 48, 52, 55-57).

Las grandes dificultades que conllevan el ajuste y seguimiento de la dieta obligan a unos rigurosos controles clínico-bioquímicos en estos pacientes. Es muy importante realizar controles periódicos del estado nutricional y de los niveles de metabolitos. El estado nutricional se valora controlando el desarrollo físico (curva ponderal y tono muscular) y los parámetros bioquímicos (proteínas totales, albúmina, balance nitrogenado) (12).

Durante el tratamiento dietético los metabolitos diagnósticos no desaparecen totalmente, solamente disminuyen respecto a los niveles del diagnóstico. Por el contrario, sí desaparecen los metabolitos producidos en las vías secundarias alternativas, como los de cetosis. Se recomienda mantener la isovalerilglicina en orina menor de $2000 \mathrm{mmol} /$ mol creatinina (variable para cada paciente). Es muy importante controlar que no aparezcan otros metabolitos como el 3-hidroxi-isovalerato, sugestivo de descompensación.

En estos pacientes sometidos a tratamiento conviene controlar los niveles en plasma y orina de aquellos aminoácidos restringidos en la dieta, para evitar niveles bajos o deficientes. También se deberán monitorear los niveles de carnitina libre (deberá ser normal) y carnitina total (deberá ser elevada).

Los controles bioquímicos en los pacientes con AIV deberán realizarse cuando estén en crisis de descompensación metabólica; a los 10-15 días tras modificación de la dieta; de manera mensual/ trimestral en niños $<2$ años con estabilidad clínica, y trimestral/semestral en niños $>2$ años con estabilidad clínica $(2,4,12)$.

\section{Revista Biomédica}

\section{CONCLUSIONES.}

Hasta el momento la población cubana no ha sido tamizada para la acidemia isovalérica por lo que la verdadera incidencia se desconoce. Debido a lo raro de este desórden las áreas de salud y los médicos tienen relativamente poca experiencia en el manejo de los mismos. Un aspecto importante es que los padres conozcan la enfermedad de su hijo, enseñarlo a darle una mejor atención a la misma y que conozcan los riesgos para sus próximos embarazos.

Se hace vital la estrecha relación de trabajo entre los clínicos y el laboratorio para el diagnóstico temprano, la pronta transferencia a un centro especializado y una mejor atención a la salud de nuestra población pediátrica.

\section{REFERENCIAS.}

1. Rainmann E, Cornejo V, Jiménez M. Aproximación al diagnóstico y tratamiento de los errores innatos del metabolismo. En: Colombo M, Cornejo V, Raimann E, editores. Errores innatos del metabolismo del niño. $2^{\mathrm{a}}$ ed. Santiago de Chile: Editorial universitaria SA; 2003. p. 4769.

2. Sweetman L, William JC. Branched Organic aciduria. En: Scriver Ch, Beaudet AL, Sly W, Valle D, editores. The metabolic and molecular bases of inherited disease. $8^{\text {th }}$ ed. New York: McGraw-Hill Inc; 2001. vol 2:2125-63.

3. Tanaka K, Budd MA, Efron ML, Isselbacher KJ. Isovaleric acidemia: a new genetic defect of leucine metabolism. Proc Natl Aca Sci USA 1966; 56:236-42.

4. Cornejo V, Raimann E. Errores innatos del metabolismo de los aminoácidos. En: Colombo M, Cornejo V, Raimann E, editores. Errores innatos del metabolismo del niño. $2^{\text {a }}$ ed. Santiago de Chile: Editorial universitaria SA; 2003. p. 71-138.

5. Ikeda Y, Tanaka K. Purification and characterization of isovaleryl Coenzyme A dehydrogenase from rat liver mitochondria. J Biol Chem 1983; 258:1077-85.

6. Ikeda Y, Keese SM, Fenton WA, Tanaka K. Biosynthesis of four rat liver mitochondrial acyl-CoA dehydrogenases: in vitro síntesis, import into mitochondria and processing of their precursors in a cell free system and in cultured cells. Arch Biochem Biophys 1987; 252:662-74. 


\section{Acidemia isovalérica}

7. Parimoo B, Tanaka K. Structural organization of the human isovaleryl-CoA dehydrogenase gene. Genomics 1993; 15:582-590.

8. Online mendelian inheritance in man: IsovalerylCoA dehydrogenase, 607036. Available from: URL: http/ /www.ncbi.nlm.nih.gov/OMIM.

9. Mohsen AW, Anderson BD, Volchenboum SL, Battaile KP, Tiffany K, Roberts D, et al. Characterization of molecular defects in isovaleryl-CoA dehydrogenase in patients with isovaleric acidemia. Biochemistry. 1998; 37: 10325-35.

10. Ensenauer R, Vockley J, Willard JM, Huey JC, Sass $\mathrm{JO}$, Edland SD, et al. A common mutation is associated with a mild, potentially asymptomatic phenotype in patients with isovaleric acidemia diagnosed by newborn screening. Am J Hum Genet. 2004; 75:1136-42.

11. Vockley J. Rogan PK, Andersen BD, Willard J, Seelan RS, Smith DI, et al. Exon skipping in IVD RNA processing in isovaleric acidemia caused by point mutations in the coding region of the IVD gene. Am J Hum Genet 2000; 66: 356-67.

12. Campistol J, Boveda MC, Couce ML, Lluch MD, Merinero B. Protocolo de diagnóstico y tratamiento de la acidemia propiónica-metilmalónica-isovalérica. An Esp de Pediatr 1997; 89:9-15.

13. Saudubray JM, Charpentier C. Clinical phenotypes: diagnosis/ algorithms. En: Scriver Ch, Beaudett AL, Sly W, Valle D, editores. The metabolic and molecular bases of inherited disease. 8th ed. New York : McGraw-Hill; 1995. vol 1. p. 327-400.

14. Wilcox WR, Cederbaum SD. Amino acid metabolism. En: Rimoin E, Connor JM, Pyeritz BR, editores. Emery and Rimoin`s. Principles and practice of medical genetics. $4^{\mathrm{a}} \mathrm{ed}$. New York: Churchill Livingtone; 2002. Vol 2. p. 2405-40.

15. Orbao T, Mpofu C, Blackensee D. Severe CNS bleeding followed by a good clinical outcome in acute neonatal form of isovaleric acidaemia. J Inher Metab Dis 1994; 17:755-6.

16. Online mendelian inheritance in man: Isovaleric Acidemia, 243500. Available from: URL: http// www.ncbi.nlm.nih.gov/OMIM.

17. Ogier H, Wendel U, Saudubray JM. Branched-chain organic acidurias. En: Fernández J, Saudubray JM, Van den
Berghe G, editores. Inborn metabolic Diseases. Berlín: Springer-Verlag; 1995. p. 207-28.

18. Tokatli A, Coskun T, Ozalp I. Isovaleric acidemia. Clinical presentation of 6 cases. Turk J Pediatr. 1998; 40: 111-9.

19. Feinstein JA, O’Brien K. Acute metabolic decompensation in an adult patient with isovaleric acidemia. South Med J. 2003; 96:500-3.

20. Kahler SG, Sherwood WG, Woolf D, Lawless ST, Zaritsky A, Bonham J, et al. Pancreatitis in patients with organic acidemias. J Pediatr. 1994; 124:239-43.

21. Tanaka K, Isselbacker KJ. The isolation and identification of $\mathrm{N}$-isovalerylglycine from urine of patients with isovaleric acidemia. J Biol Chem 1967; 242:2966-72.

22. Tanaka K, Orr JC, Isselbacher KJ. Identificaction of beta-hydroxyisovaleric acid in the urine of a patient with isovaleric acidemia. Biochim Biophys Acta 1968; 152:63841.

23. Truscott RJW, Malegan D, McCairns E, Burke D, Hick L, Sims P, et al. New metabolites in isovaleric acidemia. Clin Chim Acta 1981; 110:187-203.

24. Shigematsu Y, Kikawa Y, Sudo M, Kikuchi K, Ohta S, Okamata M. A simple method of determining 4-hydroxyisovaleric acid and its levels in a patient with isovaleric acidemia. Clin Chim Acta 1984; 138:333-6

25. Hine DG, Tanaka K. The identification and the excretion pattern of isovalerylglucoronide in the urine of patients with isovaleric acidemia. Pediatr Res 1984; 18: 508-12

26. Lehnert W. N-isovalerylalanina and $\mathrm{N}$ isovalerylsarcosine: Two new minor metabolites in isovaleric acidemia. Clin Chim Acta 1983; 134:207-12.

27. Lehnert W. Excretion of N-isovalerylglutamic acid in isovaleric acidemia. Clin Chim Acta 1981; 116:249-52.

28. Lehnert W. 3-hydroxyisoheptanoic acid: a new metabolite in isovaleric acidemia. Clin Chim Acta 1981; 113:101-3.

29. Rabier D, Parvy P, Bardet J, Saudubray JM, Kamoun P. Alloisoleucine in isovaleric acidemia. J Inher Metab Dis 1992; 15:154-5.

Vol. 17/No. 3/Julio-Septiembre, 2006 


\section{Martín-Hernández}

30. Loot du T, Erasmus E, Mienie LJ. Identification of 19 new metabolites induced by abnormal aminoacid conjugation in isovaleric acidemia. Clin Chem 2005; 51:1510-12.

31. Gibson KM, Elpeleg ON, Wappner RS. Disorders of leucine metabolism. En: Blau N, Duran M, Blaskovics ME, editors. Physician's guide to the laboratory diagnosis of metabolic diseases. $1^{\text {st }}$ ed. London: Chapman \& Hall; 1996. p. $125-44$

32. Roe CR, Millington DS, Maltby DA, Kahler SG, Bohan TP. L-carnitine therapy in isovaleric acidemia. J Clin Invest 1984; 74:2290-5

33. Chalmers RA, Roe CR, Stacey TE, Hoppel CL. Urinary excretion of l-carnitine and acylcarnitines by patients with disorders of organic acid metabolism: evidence for secondary insufficiency of l-carnitine. Pediatr Res 1984; 18:1325-8.

34. Wei CC, Lin WD, Tsai FJ, Wu JY, Peng CT, Tsai CH. Isovaleric acidemia diagnosed promptly by tandem mass spectrometry: report of one case. Acta Paediatr Taiwan 2004; 45:236-8.

35. Rashed MS. Clinical applications of tandem mass spectrometry: ten years of diagnosis and screening for inherited metabolic diseases. J Chromatogr B Biomed Sci App 2001; 758:27 -48.

36. Schulze A, Lindner M, Kohlmüller D, Olgemöller K, Mayatepek E, Hoffmann GF. Expanded newborn screening for inborn errors of metabolism by electrospray ionization-tandem mass spectrometry: Results, outcome, and implications. Pedriatics 2003; 111:1399-406

37. Lin WD, Wu JY, Lai CC, Tsai FJ, Tsai CH, Lin SP, et al. A pilot study of neonatal screening by electrospray ionization tandem mass spectrometry in Taiwan. Acta Paediatr Taiwan. 2001; 42:224-30.

38. Yamaguchi S, Koda N, Eto Y, Aoki K. Quick screening and diagnosis of organic acidemia by NMR urinanalysis. J Pediatr 1985; 106:620-2

39. Iles RA, Hind AJ, Chalmers RA: Use of proton nuclear magnetic resonance spectroscopy in detection and study of organic aciduria. Clin Chem 1985; 31:1795-801.

40. Hyman DB, Tanaka K. Isovaleryl CoA dehydrogenase activity in isovaleric acidemia fibroblasts using an improved tritium release assay. Pediatr Res 1986; 20:59-61.

41. Frerman FE, Goodman SI. Fluorometric assay of acyl-
CoA dehydrogenase in normal and mutant human fibroblasts. Biochem Med 1985; 33:38-44.

42. Tanaka K, Mandell R, Shih VE. Metabolism of $\left[1-{ }^{14} \mathrm{C}\right]$ and $\left[2-{ }^{14} \mathrm{C}\right]$ leucine in cultured skin fibroblasts from patients with isovaleric acidemia. J Clin Invest 1976; 58:164-72.

43. Tajima G, Sakura N, Yofune H, Dwi Bahagia Febriani A, Nishimura Y, Sakamoto A, et al. Establishment of a practical enzymatic assay method for determination of isovaleryl-CoA dehydrogenase activity using highperformance liquid chromatography. Clin Chim Acta 2005; 353:193-9.

44. Zschocke J, Hoffmann G. Metabolic pathways and their disorders. En: Zschocke J, Hoffmann G, editores. Vademecum metabolicum. Manual of metabolic pedriatrics. $2^{\text {nd }}$ ed. Stuttgart: Milupa GmbH \& Co. KG; 1999. p. 3556.

45. Shigematsu Y, Hata I, Nakai A, Kikawa Y, Sudo M, Tanaka Y, et al. Prenatal diagnosis of organic acidemias based on amniotic fluid levels of acylcarnitines. Pediatr Res 1996; 39(4 Pt 1):680-4.

46. Kleijer WJ, Van der Kraan M, Huijmans JGM, Van der Hauvel CMM, Jacobs C. Prenatal diagnosis of isovaleric acidaemia by enzyme and metabolite assay in the first and second trimestre. Prenat Diagn 1995; 15:527-33.

47. Hine DG, Hack AM, Goodman SI, Tanaka K. Stable isotope dilution analysis of isovalerylglycine in amniotic fluid and urine and its application for the prenatal diagnosis of isovaleric acidemia. Pediatr Res 1986; 20:222-6.

48. Cornejo V, Raimann E. Errores innatos del metabolismo de los aminoácidos. En: Colombo M, Cornejo V, Raimann E, editores. Errores innatos del metabolismo del niño. $2^{\mathrm{a}}$ ed. Santiago de Chile: Editorial universitaria SA; 1999. p. 59-112.

49. Low LCK. Inborn errors of metabolism: clinical approach and management. HKMJ 1996; 2:274-81.

50. Schaefer F, Straube E, Oh J, Mehls O, Mayatepek E. Dialysis in neonates with inborn errors of metabolism. Nephrol Dial Tranplant 1999; 14:910-8.

51. Ogier H. Management of the acutely sick once they arrive in hospital. En: Portnoi P, Mallery C, Fogg D, editores. Dietary Management of metabolic disease. Royal college of Physicians, London. Inborn Error Review Series No. 12; 2002. p. 5-8.

\section{Revista Biomédica}


52. Ramos BE, Pascual M. Tratamiento dietético de las enfermedades metabólicas. Inf Ter Sist Nac Salud 2005; 29: 81-95.

53. Leonard JV, Daishp HP, Naughten ER, Barlett K. The management and long-term outcome of organic acidemias. J Inher Metab Dis 1994; 17(Suppl 1):13-7.

54. Fries MH, Rinaldo P, Schmidt-Sommerfeld E, Jurecki $\mathrm{E}$, Packman S. Isovaleric acidemia: response to a leucine load after three weeks of supplementation with glycine, Lcarnitine, and combined glycine-carnitine therapy. J Pediatr 1996; 129:449-52.

55. Itoh T, Ito T, Ohba S, Sugiyama N, Mizuguchi K, Yamaguchi S, Kidouchi K. Effect of carnitine administration on glycine metabolism in patients with isovaleric acidemia: significance of acetylcarnitine determination to estimate the proper carnitine dose. Tohoku J Exp Med 1996; 179:1019.

56. Mayatepek E, Kurczynski TW, Hoppel CL. Long-term L-carnitine treatment in isovaleric acidemia. Pediatr Neurol. 1991; 7:137-40.

57. Acosta P, Yannicelli S. Nutrition support of infant, children and adults with disorders of leucine catabolism. En: Sproat K, editor. The ross metabolic formula system, nutrition support protocols. $3^{\text {th }}$ ed. New York: Ross products division; 1997. p. 109-29. 